\title{
Telmisartan is effective to ameliorate metabolic syndrome in rat model - a preclinical report
}

This article was published in the following Dove Press journal: Diabetes, Metabolic Syndrome and Obesity:Targets and Therapy

\author{
Kai-Chun Cheng ${ }^{1, *}$ \\ Yingxiao $\mathrm{Li}^{1,2, *}$ \\ Wei-Ting Chang ${ }^{2,3}$ \\ Feng Yu Kuo ${ }^{4}$ \\ Zhih-Cherng Chen ${ }^{3,5}$ \\ Juei-Tang Cheng ${ }^{2,6}$ \\ 'Department of Psychosomatic \\ Internal Medicine, Kagoshima \\ University Graduate School of \\ Medical and Dental Sciences, \\ Kagoshima 890-8544, Japan; \\ 2Department of Medical Research, \\ Chi-Mei Medical Center, Yong \\ Kang, Tainan City 7l003, Taiwan; \\ ${ }^{3}$ Department of Cardiology, Chi-Mei \\ Medical Center, Yong Kang, Tainan City \\ 7I003, Taiwan; ${ }^{4}$ Cardiovascular Center, \\ Kaohsiung Veterans General Hospital, \\ Kaohsiung City 81362, Taiwan; \\ ${ }^{5}$ Department of Pharmacy, Chia Nan \\ University of Pharmacy and Science, \\ Jean-Tae, Tainan City 7I70I, Taiwan; \\ ${ }^{6}$ Institute of Medical Sciences, Chang \\ Jung Christian University, Gueiren, \\ Tainan City 7II0I, Taiwan
}

*These authors contributed equally to this work

Correspondence: Juei-Tang Cheng Institute of Medical Sciences, Chang Jung Christian University, No. I, Changda Road, Gueiren, Tainan City 7II0I, Taiwan

Tel +886625I 7864

E-mail jtcheng@mail.cjcu.edu.tw
Background: Metabolic syndrome (MS) is known to be associated with hypertension, insulin resistance, and dyslipidemia, and it raises the risk for cardiovascular diseases and diabetes mellitus. Telmisartan is used in clinic as an angiotensin II receptor blocker and it is also identified as activating peroxisome proliferator-activated receptors $\delta$ (PPAR $\delta$ ). Activation of PPAR $\delta$ produced beneficial effects on fatty acid metabolism and glucose metabolism. This study aims to investigate the effects of telmisartan on the modulation of MS in rats fed a high-fat/high-sodium diet. Methods: Rats were fed with a high-fat/high-sodium diet and received injections of streptozotocin at low dose to induce MS. Then, rats with MS were treated with telmisartan. The weight, glucose tolerance, and insulin sensitivity were measured. The lipid profiles were also obtained. The weights of retroperitoneal and epididymal fat pads were determined. The role of PPAR $\delta$ in telmisartan treatment was identified in rats pretreated with the specific antagonist GSK0660.

Results: The results showed that telmisartan, but not losartan, significantly reduced plasma glucose and plasma insulin, and improved insulin resistance in rats with MS. Telmisartan also decreased blood pressure and lipids more significantly than losartan. Moreover, GSK0660 effectively reversed the effects of telmisartan in the MS rats. In the MS group, telmisartan activated PPAR $\delta$ to enhance the levels of phosphorylated GLUT4 in muscle or the expression of phosphoenolpyruvate carboxykinase (PEPCK) in the liver, which was also abolished by GSK0660. Telmisartan is useful to ameliorate hypertension and insulin resistance in rats with MS. Telmisartan improves the insulin resistance through increased expression of GLUT4 and down-regulation of PEPCK via PPAR $\delta$-dependent mechanisms.

Conclusion: Telmisartan has been proven to ameliorate MS, particularly in the prediabetes state. Therefore, telmisartan is suitable to develop for the management of MS in clinics.

Keywords: metabolic syndrome, telmisartan, PPAR $\delta$, GSK0660, diet

\section{Introduction}

Metabolic syndrome (MS) is a cluster of risk factors for metabolic abnormalities and cardiovascular disease. It includes abdominal obesity, dyslipidemia, hypertension, and hyperglycemia. ${ }^{1}$ Prevalence of MS is rapidly increasing worldwide. ${ }^{2}$ Approximately 31 $\%$ of the world's adult population is estimated to have MS. ${ }^{3}$ Moreover, MS is associated with a 2.5 -fold increase in cardiovascular- and diabetes-related mortalities. ${ }^{4}$

Diet is a potential factor that could be responsible for the rise in MS and the associated cardiovascular pathologies. ${ }^{5}$ The diet pattern in Western countries is generally characterized by high intake of carbohydrates and saturated fat. The increase in calorific intake has been associated with many diet-induced complications, including MS, 
cardiovascular diseases, and nonalcoholic fatty liver disease. High dietary fat intake is associated with oxidative stress and an activation of the proinflammatory transcription factors. ${ }^{6}$

High salt intake is also a significant environmental factor and is strongly associated with high blood pressure (BP). It has been previously indicated that essential hypertension is frequently related to insulin resistance and compensatory hyperinsulinemia. ${ }^{7} \mathrm{MS}$ patients also exhibit enhanced $\mathrm{BP}$ in response to sodium intake. ${ }^{8}$ Insulin resistance could activate the renin-angiotensin system (RAS) by increasing the expression of angiotensinogen, angiotensin II (AT2), and angiotensin receptor (AR), which may contribute to the development of hypertension. ${ }^{9}$ It has been recently discovered that adipocytes also produce aldosterone in response to AT2. ${ }^{10}$

The peroxisome proliferator-activated receptor $\delta$ (PPAR $\delta$ ) is a transcription factor that belongs to the superfamily of nuclear receptors. ${ }^{11}$ Activation of PPAR $\delta$ has beneficial effects on fatty acid and glucose metabolism. ${ }^{12}$ Moreover, PPAR $\delta$ could enhance fatty acid $\beta$-oxidation and attenuate MS. ${ }^{13}$ PPAR $\delta$ activation could prevent obesity and exert protective effects on hypertension and on the early manifestations of atherosclerosis in high-fat (HF) diet-fed mice. ${ }^{14}$

Telmisartan, an AR blocker (ARB), has the highest affinity for AT2 receptors among the available ARBs. ${ }^{15}$ Telmisartan has a profound role in the improvement of glucose homeostasis in skeletal muscle, which is associated with activation of PPAR $\delta .{ }^{16}$ Several studies have revealed that telmisartan improves insulin sensitivity in patients with hypertension or the early stages of diabetes mellitus. ${ }^{17,18}$

Many animal models were used to study disorders of $\mathrm{MS}^{19}$ in a manner to mimic the major signs of MS. In the induction of animal models, various approaches were applied in rodents including dietary manipulation, genetic modification, and drugs. ${ }^{19}$ However, limitations of each model were observed. Dietary approaches included the use of a single type of diet or a combination of diets, such as high-fructose, ${ }^{20}$ $\mathrm{HF},{ }^{21}$ high-fructose/ $\mathrm{HF}^{22}$ which usually affects the wholebody metabolism, but the effects is limited ${ }^{23}$ and the symptom did not include hypertension. The genetic models of MS included leptin-deficient (ob/ob) or leptin receptor-deficient $(\mathrm{db} / \mathrm{db})$ mice. Unlike humans with MS, ob/ob mice did not develop dyslipidaemia, ${ }^{24}$ and both ob/ob and $\mathrm{db} / \mathrm{db}$ mice did not show hypertension. ${ }^{25,26}$ Although HF fed, spontaneously hypertensive rats show some symptoms of MS, they have genetically induced, rather than diet-induced, hypertension. ${ }^{27}$ Models of drug-induced MS include glucocorticoid- induced ${ }^{28}$ and antipsychotic-induced ${ }^{29}$ models, which seem more suitable for the research of specific diseases. In this study, we established a MS model based on environmental effects, which promoted blood glucose, blood pressure, and blood fat using the HF, high-sodium (HS) diet intake and a low-dose of streptozotocin (STZ) injection. The main aim of this study was to investigate the effects of telmisartan on insulin resistance, hyperlipidemia, and hypertension in rats with MS.

\section{Methods}

\section{Animals}

Male Sprague Dawley rats weighing 180-220 g were obtained from the National Animal Center (Taipei, Taiwan) and maintained in the animal center of Chi Mei Medical Center (Tainan, Taiwan). The animals were housed two rats per cage on a 12/12-hour light/dark cycle at a constant temperature $\left(24^{\circ} \mathrm{C} \pm 1{ }^{\circ} \mathrm{C}\right)$ and humidity $(60 \% \pm 10 \%)$. This project was approved by the Institutional Animal Care and Use Committee of Chi Mei Medical Center (No. 105110330). The Guide for the Care was referred to during this study.

\section{Rat model with MS}

The rats were randomly fed either standard rat chow (13.43\% kcal as fat; TestDiet ${ }^{\mathbb{R}}$; Richmond, IN, USA) or HF/HS diet for 8 weeks. Custom HF diets $\left(60 \%\right.$ kcal as fat; LabDiet ${ }^{\circledR}$; St Louis, MO, USA) were applied to prepare a HF/ HS (4\% $\mathrm{NaCl})$ diet. ${ }^{30}$ All rats freely received normal tap water.

After an 8-week feeding of a $\mathrm{HF} / \mathrm{HS}$ diet, rats were starved for 12 hours then injected intraperitoneally with STZ at low dose $(30 \mathrm{mg} / \mathrm{kg})^{31}$ and were continued to be fed the same diet during the experiments. After 7 days of STZ injections, the rats had hyperglycemia $(>200 \mathrm{mg} / \mathrm{dL})$, hyperlipidemia (total cholesterol [TC] $>110 \mathrm{mg} / \mathrm{dL}$ and triglyceride $[\mathrm{TG}]>150 \mathrm{mg} / \mathrm{dL}$ ), an increase in body weight ( $8 \%$ of initial weight) or mean arterial $\mathrm{BP}>130 \mathrm{mmHg}$, and a marked decrease in high-density lipoprotein (HDL) cholesterol $(<35 \mathrm{mg} / \mathrm{dL})$ that were used to confirm the development of $\mathrm{MS}^{32}$ Rats with MS were allowed the $\mathrm{HF} / \mathrm{HS}$ diet until the end of the study.

\section{Treatment protocols}

Once MS occurred, models and controls were treated by oral gavage with telmisartan $(8 \mathrm{mg} / \mathrm{kg} /$ day; Boehringer Ingelheim, Ingelheim am Rhein, Germany $)^{10}$ or losartan $(8 \mathrm{mg} / \mathrm{kg} /$ day; Zydus Pharmaceuticals, Pennington, NJ, USA) for 4 weeks. Moreover, PPAR $\delta$ antagonist GSK0660 (10 mg/kg; Sigma- 
Aldrich Co., St Louis, MO, USA) was intraperitoneally injected 30 minutes before telmisartan administration.

Food and water intake were measured daily. Body weight was monitored weekly. BP was determined at week 9 (before drug treatment) and week 13 (the end of 4-week periods of the drug treatment) using the tail-cuff method with a sphygmomanometer (Muromachi Kikai Co., Ltd., Tokyo, Japan). ${ }^{33}$

At week 14, insulin tolerance tests (ITTs) were performed in the rats fasting overnight. According to a previous report, ${ }^{34}$ rats were intraperitoneally injected with $0.75 \mathrm{IU} / \mathrm{kg}$ of regular insulin. Blood was collected from the tail vein of rats under anesthesia before injection and after 15, 30, 60, 90, and 120 minutes.

At the end of the study, livers and soleus muscles were collected from the sacrificed rats. The weight of retroperitoneal and epididymal fat pads were also measured. All samples were immediately frozen in liquid nitrogen and kept at $-80^{\circ} \mathrm{C}$ for further assays.

\section{Biochemical measurements}

Blood samples were collected from the tail vein of rats that were anesthetized with sodium pentobarbital ( $50 \mathrm{mg} / \mathrm{kg}$, i.p.) and all efforts were made to minimize the animals' suffering. Blood glucose concentration was measured ${ }^{35}$ using commercial kits (Wako Pure Chemical Industries, Ltd., Osaka, Japan). Serum insulin concentrations were also measured using commercialized enzyme-linked immunosorbent assay kits (Mercodia AB, Uppsala, Sweden). The following formula was used to calculate the homeostasis model assessment for insulin resistance (HOMA-IR): (fasting insulin $[\mu \mathrm{U} / \mathrm{mL}] \times$ fasting glucose $[\mathrm{mg} / \mathrm{dL}]) / 405$. Additionally, the area under the curve was evaluated for the glucose concentrations determined at $0,30,60,90$, and 120 minutes. The lipid profile, including concentrations of TC, TG, and HDL, was estimated using laboratory kit reagents (Randox Laboratories, Crumlin, UK). The low-density lipoprotein (LDL) levels were then calculated using Frieldwann's equation.

\section{Western blotting analysis}

The Western blotting analysis was was performed according to the previous method. ${ }^{52}$ Total protein lysates were extracted in lysis buffer (1\% Triton X-100, $150 \mathrm{mM} \mathrm{NaCl}, 10 \mathrm{mM}$ Tris [pH 7.5], and $5 \mathrm{mM}$ ethylenediaminetetraacetic acid), containing a protease and phosphatase inhibitor cocktail (Sigma-Aldrich Co.). The protein concentration was determined with the BCA Protein Assay Kit (Thermo Fisher Scientific, Waltham, MA, USA). The following primary antibodies were used at $4^{\circ} \mathrm{C}$ overnight: anti-PPAR $\delta(1: 1000)$ and anti-GLUT4 (1:1000) (Abcam, Cambridge, UK); and anti-phosphoenolpyruvate carboxykinase (anti-PEPCK) (1:1000) (EMD Millipore, Billerica, MA, USA) was used as an internal control. The next day, the blots were incubated in the secondary antibodies at room temperature for 1 hour. Protein bands were visualized using the enhanced chemiluminescence kit (PerkinElmer Inc., Boston, MA, USA). The optical densities of the bands were determined using software (Gel-Pro ${ }^{\circledR}$ Analyzer Version 4.0 software; Media Cybernetics Inc., Rockville, MD, USA).

\section{Statistical analyses}

All results are provided as mean \pm SEM of each group. All statistical analyses were carried out by SPSS, Version 21 (IBM Corporation, Armonk, NY, USA). Differences between the two groups were determined by two-way repeated measures ANOVA. For comparisons between two independent groups, a Student's $t$-test was used. Significance was accepted at $p<0.05$.

\section{Results}

\section{Telmisartan ameliorated hyperglycemia and insulin resistance in MS rats}

Rats fed the HF/HS diet for 8 weeks followed by an injection of STZ $(30 \mathrm{mg} / \mathrm{kg})$ developed MS because they became hyperphagic, obese, hyperlipidemia, insulin resistant, and hypertensive. The MS group displayed moderate glucose intolerance characterized by a 2.0 -fold increase in fasting serum glucose and 2.2-fold increase in HOMA-IR index. In ITT experiments, the MS group showed higher glucose levels in serum that were improved by telmisartan treatment compared with the normal control group.

Blood glucose was significantly reduced in MS rats treated with telmisartan compared with the vehicle-treated MS group $(-28 \%, P<0.05)$ (Figure 1A). Similarly, the HOMA-IR was significantly attenuated in the telmisartantreated group $(-29 \%, P<0.05)$. However, losartan did not affect the glucose levels in MS rats (Figure $1 \mathrm{C}-\mathrm{E}$ ).

\section{Effects of telmisartan on body weight, food intake and white adipose mass in MS rats}

The MS group showed a $14 \%$ increase in body weight compared with the control group. Also, the MS group significantly increased retroperitoneal fat mass and epididymal fat mass in a different way to the control group (Table 1). In addition, the 

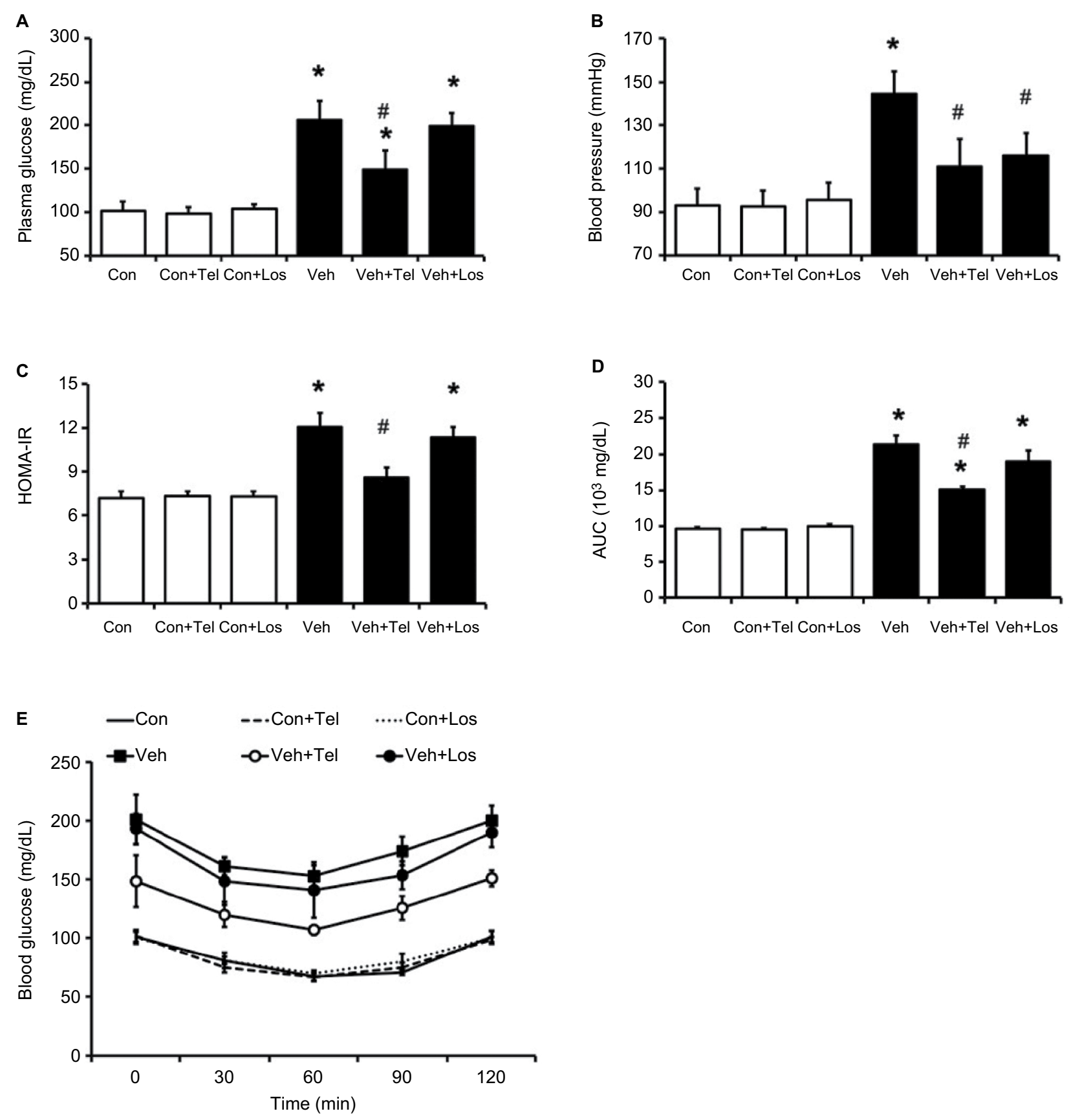

Figure I Effects of telmisartan or losartan on MS rats.

Notes: The change in (A) blood glucose; (B) mean arterial blood pressure; (C) HOMA-IR; (D) AUC of HOMA-IR; and (E) ITT in normal rats and MS rats after 4 weeks of experimental period. Con (white column), SD rats fed normal chow diet; Con+Tel (white column), SD rats fed normal chow diet and administered with telmisartan (8mg/kg); Con+Los (white column), SD rats fed normal chow diet and administered with losartan (8mg/kg); Veh (black column), MS rats received with vehicle; Veh+Tel (black column), MS rats received administered with telmisartan $(8 \mathrm{mg} / \mathrm{kg})$ for 4 weeks; Veh+Los (black column), MS rats received administered with losartan (8mg/kg) for 4 weeks. Data are expressed as mean \pm SEM $(n=6)$. ${ }^{P} P<0.05$ vs Con; ${ }^{*} P<0.05$ vs Veh.

Abbreviations: AUC, area under the curve; Con, control; HOMA-IR, homeostatic model assessment for insulin resistance; ITT, insulin tolerance test; Los, losartan; MS, metabolic syndrome; SD, Sprague Dawley; Veh, vehicle; Tel, telmisartan.

average daily food intake and water intake were elevated in MS groups more markedly than in the control group (Table 2).

Telmisartan significantly reduced the rise in body weight of MS rats compared with the vehicle-treated MS rats. Retroperitoneal fat mass and epididymal fat mass were also noted to be reduced by telmisartan in the MS group at the end of experiments. However, a similar change was not observed in MS rats administered with losartan. Additionally, telmisartan or losartan did not influence body weight or fat mass in rats that received normal chow diet (Table 1). 
Table I Effects of telmisartan (Tel) or losartan (Los) on the metabolic parameters in normal rat and MS rats

\begin{tabular}{|l|l|l|l|l|l|l|}
\hline & Con & Con+Tel & Con+Los & MS +Veh & MS +Tel & MS +Los \\
\hline TG $(\mathrm{mg} / \mathrm{dL})$ & $70.5 \pm 7.21$ & $72.88 \pm 8.13$ & $73.75 \pm 7.05$ & $131.00 \pm 9.74^{*}$ & $105.63 \pm 8.43^{* \#}$ & $122.25 \pm 8.3 I^{*}$ \\
\hline $\mathrm{TC}(\mathrm{mg} / \mathrm{dL})$ & $57.75 \pm 4.53$ & $57.63 \pm 5.45$ & $58.63 \pm 4.66$ & $74.13 \pm 5.59^{*}$ & $67.13 \pm 5.17^{\#}$ & $70.63 \pm 6.55^{*}$ \\
\hline $\mathrm{HDL}(\mathrm{mg} / \mathrm{dL})$ & $25.00 \pm 2.39$ & $25.75 \pm 2.19$ & $25.88 \pm 1.73$ & $20.50 \pm 1.3 I^{*}$ & $24.25 \pm 2.66^{\#}$ & $21.25 \pm 1.75^{*}$ \\
\hline $\mathrm{LDL}(\mathrm{mg} / \mathrm{dL})$ & $17.90 \pm 4.82$ & $17.30 \pm 6.07$ & $18.00 \pm 4.77$ & $27.43 \pm 6.28^{*}$ & $18.55 \pm 7.94^{\#}$ & $24.93 \pm 5.96^{*}$ \\
\hline Retroperitoneal fat mass $(\mathrm{g})$ & $4.54 \pm 0.75$ & $4.49 \pm 0.66$ & $4.71 \pm 0.45$ & $6.78 \pm 0.75^{*}$ & $5.66 \pm 0.78^{\#}$ & $6.71 \pm 0.55^{*}$ \\
\hline Epididymal fat mass $(\mathrm{g})$ & $4.23 \pm 0.53$ & $4.33 \pm 0.69$ & $4.58 \pm 0.57$ & $7.21 \pm 0.95^{*}$ & $5.98 \pm 0.96^{* \#}$ & $6.44 \pm 0.56^{*}$ \\
\hline Body weight (g) & $272.63 \pm 10.0 \mathrm{I}$ & $276.5 \pm 7.83$ & $279.75 \pm 8.71$ & $311.38 \pm 13.70^{*}$ & $289.25 \pm 6.84^{\#}$ & $314.13 \pm 16.25^{*}$ \\
\hline $\begin{array}{l}\text { The increase of body weight } \\
\text { (Compared with Con,\%) }\end{array}$ & $\approx 0$ & $\approx 0$ & $\approx 0$ & $14.36 \pm \mathrm{I} .72^{*}$ & $4.43 \pm 1.55^{\#}$ & $15.14 \pm 1.20^{*}$ \\
\hline
\end{tabular}

Notes: Control (Con), SD rats fed normal chow diet; Metabolic Syndrome (MS), SD rats were induced MS model as described in methods; Vehicle (Veh), MS rats received vehicle. Rats were daily administered with telmisartan $(8 \mathrm{mg} / \mathrm{kg})$ or losartan $(8 \mathrm{mg} / \mathrm{kg})$ for 4 weeks. Data shown mean $\pm S E M(n=6)$. ${ }^{*} P<0.05$ vs. Con; ${ }^{*} P<0.05$ vs. Veh. Abbreviation: SD, Sprague Dawley; Con, control; Los, losartan; MS, metabolic syndrome; Veh, vehicle; Tel, telmisartan.

Table 2 Effects of telmisartan or losartan on food intake and water intake in normal rats and MS rats

\begin{tabular}{|l|l|l|l|l|l|l|}
\hline & Con & Con+Tel & Con+Los & MS+Veh & MS+Tel & Con+Los \\
\hline Food intake (g/day) & $36.88 \pm 2.47$ & $39.13 \pm 3.76$ & $38.38 \pm 1.89$ & $47.88 \pm 1.35^{*}$ & $49.50 \pm 1.77^{*}$ & $48.75 \pm 4.20$ \\
\hline Water intake (g/day) & $30.88 \pm 2.47$ & $30.75 \pm 2.25$ & $29.25 \pm 1.84$ & $41.88 \pm 3.489$ & $43.50 \pm 5.13^{*}$ & $45.75 \pm 4.49$ \\
\hline
\end{tabular}

Notes: Average daily food and water intakes were calculated. Con, SD rats fed normal chow diet; MS, MS rat model using SD rats as described in Methods; Veh, MS rats received vehicle. Rats were administered daily with telmisartan $(8 \mathrm{mg} / \mathrm{kg})$ or losartan $(8 \mathrm{mg} / \mathrm{kg})$ for 4 weeks. Data are expressed as mean $\pm S E M(n=6)$. $* P<0.05$ vs Con; ${ }^{\# P}<0.05$ vs Veh.

Abbreviations: Con, control; Los, losartan; MS, metabolic syndrome; SD, Sprague Dawley; Tel, telmisartan; Veh, vehicle.

\section{Telmisartan improved lipid metabolism in MS rats}

Lipids in normal control or MS rats treated with telmisartan or losartan shown in Table 1. Significant increases in TC, TG, HDL, and LDL cholesterol concentrations were observed in MS rats.

Telmisartan attenuated the plasma TC, TG and LDL levels significantly and increased the HDL levels. However, losartan produced a little improvement in the lipids in MS rats compared with telmisartan-treated MS rats.

\section{Effects of telmisartan and losartan on BP}

Mean arterial BP was significantly increased in MS rats and mild hypertension was observed compared with the control group ( $144.3 \pm 10.7$ vs $92.9 \pm 7.9 \mathrm{mmHg}, P<0.05)$. A significant reduction in $\mathrm{BP}$ was observed in MS rats treated with telmisartan or losartan (telmisartan: $110 \pm 12.7 \mathrm{mmHg}$ and losartan: $115.8 \pm 10.6 \mathrm{mmHg}$ ) compared with those not treated $(144.3 \pm 10.7 \mathrm{mmHg}, P<0.05)$ (Figure 1B).

\section{PPAR $\delta$ antagonist inhibited the effects of telmisartan in MS rats}

To investigate the role of PPAR $\delta$ in the effects of telmisartan in MS rats, the selective PPAR $\delta$ antagonist GSK0660 was pretreated with telmisartan.
GSK0660 significantly reduced the beneficial effects of telmisartan in blood glucose (from $153.0 \pm 17.1 \mathrm{mg} / \mathrm{dL}$ to $185.6 \pm 14.2 \mathrm{mg} / \mathrm{dL}, P<0.05$ ) (Figure $2 \mathrm{~A}$ ). Additionally, TG and TC levels in the telmisartan-treated MS group were reversed markedly (Table 3). Moreover, pretreatment with GSK0660 reversed the reduction of HOMA-IR index and the improvement of ITT, which were induced by telmisartan in MS rats $(P<0.05)$ (Figure $2 \mathrm{C}-\mathrm{E})$. In addition, the BP-lowering effect of telmisartan was also inhibited by GSK0660; GSK0660 reversed BP from $137 \pm 1 \mathrm{mmHg}$ to $152 \pm 1 \mathrm{mmHg}$ $(P<0.05)$ showing $\sim 30 \%$ recovery in the telmisartan-treated MS group (Figure 2B). Administration of GSK0660 also reversed the glucose and lipid profiles modified by telmisartan in MS rats but not in the control group. In addition, GSK0660 also reversed the body weight and adipose mass in MS rats attenuated by telmisartan (Table 3).

Telmisartan ameliorates MS through PPAR $\delta$-dependent mechanisms in liver and skeletal muscle

The protein levels of GLUT4 in skeletal muscle and PEPCK in the liver of MS rats were determined. As shown in Figure 3A, the decreased GLUT4 and PPAR $\delta$ expression in the soleus muscle of MS rats were reversed by telmisartan. Additionally, the increased hepatic PEPCK level in MS rats was also markedly reduced by telmisartan (Figure 3B). But similar changes were not observed in losartan-treated MS 
A

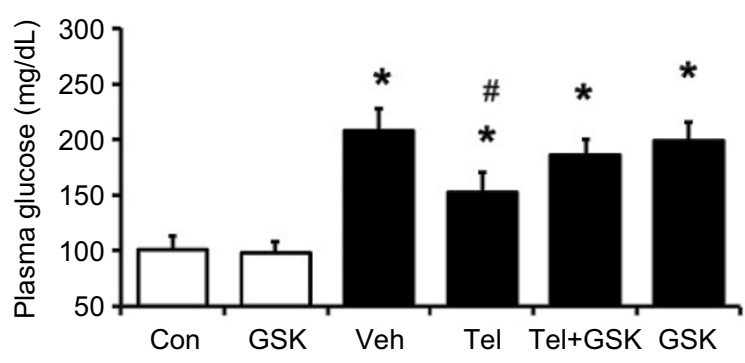

C

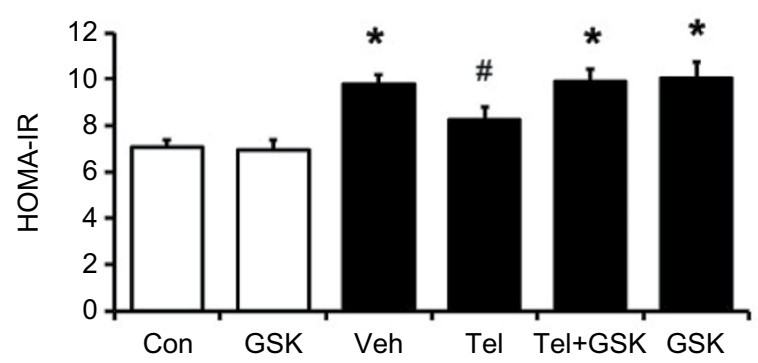

$\mathbf{E}$

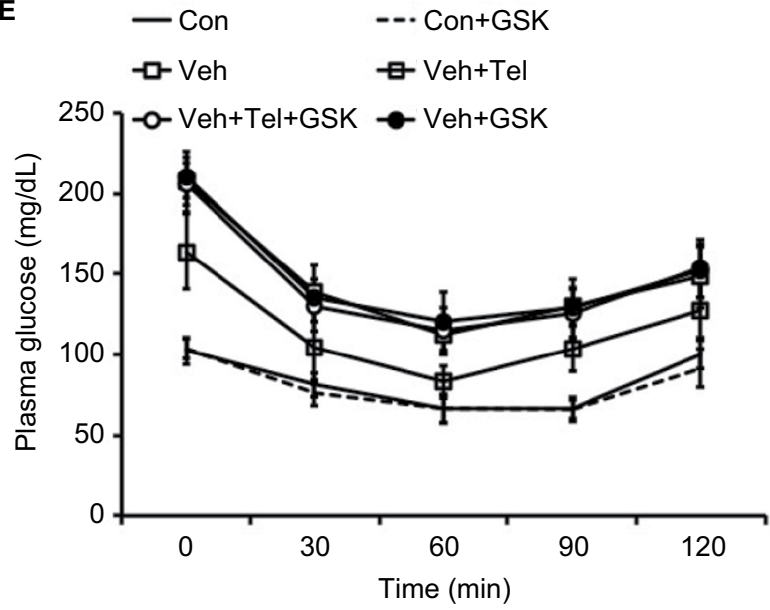

B

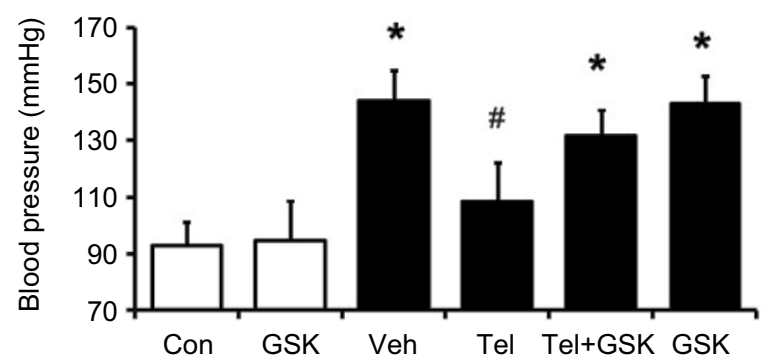

D

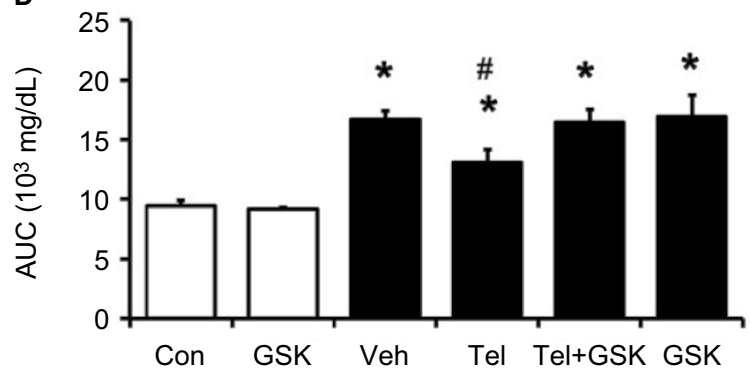

Figure 2 PPAR $\delta$ antagonist GSK0660 inhibited the effects induced by chronic telmisartan treatment in MS rats.

Notes: The changes in (A) blood glucose; (B) mean arterial blood pressure; (C) HOMA-IR; (D) AUC of HOMA-IR; and (E) ITT. Con (white column), SD rats fed normal diet; Con+Tel (white column), SD rats fed normal diet and administered with telmisartan $(8 \mathrm{mg} / \mathrm{kg})$; Veh (black column), MS rats received telmisartan (8mg/kg) for 4 weeks and GSK0660 were pretreated 30 minutes before telmisartan treatment; Veh+GSK (black column) MS rats received administered with vehicle and GSK0660 for 4 weeks. Data are expressed as mean $\pm \operatorname{SEM}(n=6)$. $* P<0.05$ vs Con; ${ }^{\#}<<0.05$ vs Veh.

Abbreviations: AUC, area under the curve; Con, control; HOMA-IR, homeostatic model assessment; ITT, insulin tolerance test; MS, metabolic syndrome; PPAR peroxisome proliferator-activated receptor delta; SD, Sprague Dawley; Tel, telmisartan; Veh, vehicle.

rats. Moreover, the effects of telmisartan were attenuated by GSK0660 in MS rats (Figure 4). Therefore, the results suggested that the effects of telmisartan in liver and skeletal muscle were PPAR $\delta$ dependent.

\section{Discussion}

A rat model was successfully developed for MS using the modified diet and pancreatic toxin described in this study.
Male SD rats received a $\mathrm{HF} / \mathrm{HS}$ diet for 8 weeks, followed by a low-dose STZ $(30 \mathrm{mg} / \mathrm{kg})$ injection. The MS rats showed insulin resistance, impaired glucose tolerance, obesity, dyslipidemia, and hypertension. Moreover, this MS model mimics the main changes that occur in humans.

In this study, we found that telmisartan improved glucose and/or lipid profiles in MS rats through PPAR $\delta$ activation. Telmisartan significantly decreased the plasma insulin 
A Con Con+Tel Con+Los Veh Veh+Tel Veh+Los
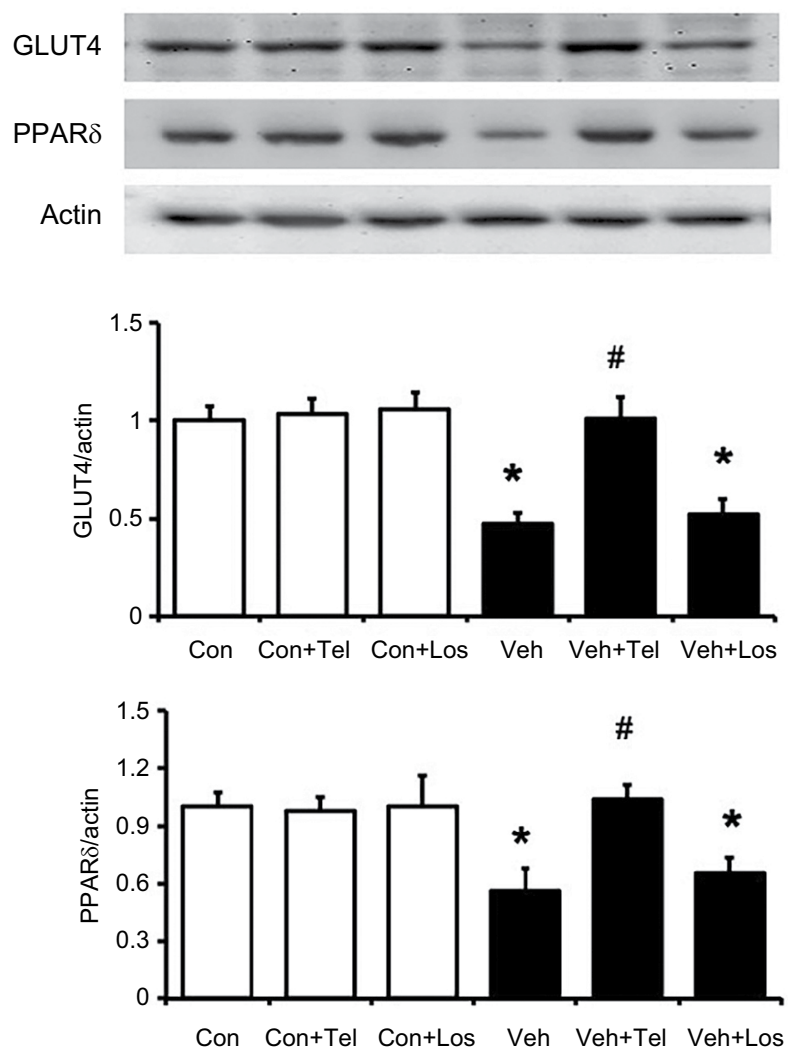

B

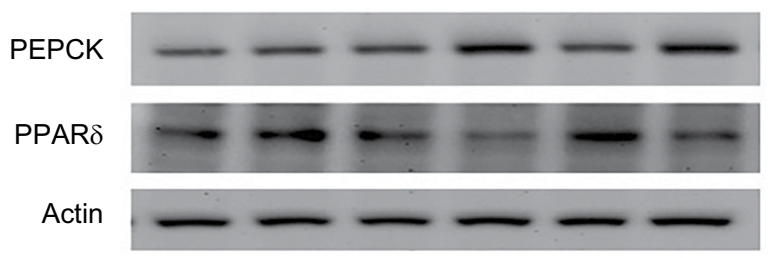

Figure 3 Effects of telmisartan or losartan on PPAR $\delta$ and related signal expression in skeletal muscle and liver.

Notes: (A) Representative immunoblots are shown in the upper part of the figure, and the relative expression levels of GLUT4 and PPAR $\delta$ expression in soleus muscle are indicated in the lower. (B) Representative immunoblots are shown in the upper part of the figure, and the relative expression levels of PEPCK and PPAR $\delta$ in liver are indicated in the lower. Con (white column), SD rats fed normal diet; Con+Tel (white column), SD rats fed normal diet and administered with telmisartan (8mg/kg); Con+Los (white column), SD rats fed normal diet and administered with losartan $(8 \mathrm{mg} / \mathrm{kg}$ ); Veh (black column), MS rats received with vehicle; Veh+Tel (black column), MS rats received administered with telmisartan $(8 \mathrm{mg} / \mathrm{kg})$ for 4 weeks; Veh+Los (black column), MS rats received administered with losartan (8mg/kg) for 4 weeks. Data are expressed as mean \pm SEM $(n=4) . * P<0.05$ vs Con; ${ }^{*} P<0.05$ vs Veh.

Abbreviations: Con, control; PEPCK, phosphoenolpyruvate carboxykinase; PPAR $\delta$, peroxisome proliferator-activated receptor delta; Los, losartan; MS, metabolic syndrome; SD, Sprague Dawley; Tel, telmisartan; Veh, vehicle.

Table 3 GSK0660 inhibited the effects of telmisartan on various metabolic parameters in MS rats

\begin{tabular}{|c|c|c|c|c|c|c|}
\hline Parameters & Con & Con+GSK & MS+Veh & MS+Tel & MS+Tel+GSK & MS+GSK \\
\hline TG $(\mathrm{mg} / \mathrm{dL})$ & $70.50 \pm 7.21$ & $72.38 \pm 9.68$ & $131.00 \pm 9.07 *$ & $105.63 \pm 8.43^{* \#}$ & $135.88 \pm 10.80 *$ & $138.00 \pm 12.33^{*}$ \\
\hline $\mathrm{TC}(\mathrm{mg} / \mathrm{dL})$ & $57.75 \pm 4.53$ & $59.63 \pm 4.21$ & $74.13 \pm 5.59 *$ & $67.13 \pm 5.17^{\#}$ & $77.13 \pm 8.48^{*}$ & $77.25 \pm 8.60 *$ \\
\hline $\mathrm{HDL}(\mathrm{mg} / \mathrm{dL})$ & $25.00 \pm 2.39$ & $24.25 \pm 2.38$ & $20.50 \pm I .3 I^{*}$ & $24.25 \pm 2.66^{\#}$ & $21.38 \pm 1.85^{*}$ & $20.63 \pm 2.83 *$ \\
\hline $\mathrm{LDL}(\mathrm{mg} / \mathrm{dL})$ & $17.90 \pm 4.82$ & $19.90 \pm 6.15$ & $27.43 \pm 6.28 *$ & $18.55 \pm 7.94^{\#}$ & $28.58 \pm 10.53^{*}$ & $29.03 \pm 9.54 *$ \\
\hline Retroperitoneal fat mass (g) & $4.54 \pm 0.75$ & $4.62 \pm 0.59$ & $6.78 \pm 0.75 *$ & $5.66 \pm 0.78^{\#}$ & $7.0 \mathrm{I} \pm 0.65^{*}$ & $6.85 \pm 0.42 *$ \\
\hline Epididymal fat mass (g) & $4.23 \pm 0.53$ & $4.3 I \pm 0.73$ & $7.21 \pm 0.95^{*}$ & $5.98 \pm 0.96^{* \#}$ & $6.92 \pm 0.58 *$ & $6.44 \pm 0.56^{*}$ \\
\hline Body weight $(\mathrm{g})$ & $272.63 \pm 10.01$ & $268.4 \pm 8.83$ & $311.38 \pm 13.70 *$ & $289.25 \pm 6.84^{\#}$ & $320.5 \mathrm{I} \pm 13.3 \mathrm{I} *$ & $318.87 \pm 14.58^{*}$ \\
\hline $\begin{array}{l}\text { The increase of body } \\
\text { weight (compared with Con, \%) }\end{array}$ & $\approx 0$ & $\approx 0$ & $14.36 \pm 1.72 *$ & $4.43 \pm 1.55^{\#}$ & $16.19 \pm\left. 2.5\right|^{*}$ & $15.74 \pm 2.32^{*}$ \\
\hline
\end{tabular}

Notes: Con, SD rats fed normal chow diet; MS, MS rat model using SD rats as described in Methods; Veh, MS rats received the vehicle. Rats were administered with telmisartan $(8 \mathrm{mg} / \mathrm{kg})$ or losartan $(8 \mathrm{mg} / \mathrm{kg})$ for 4 weeks. GSK0660 were pretreated $30 \mathrm{~min}$ before telmisartan administration. Data are expressed as mean \pm SEM $(\mathrm{n}=6)$. $* P<0.05$ vs Con; ${ }^{*} P<0.05$ vs Veh.

Abbreviations: Con, control; HDL, high-density lipoprotein; LDL, low-density lipoprotein; MS, metabolic syndrome; SD, Sprague Dawley; TC, total cholesterol; Tel, telmisartan; TG, triglyceride; Veh, vehicle.

and markedly reduced plasma glucose. Telmisartan also alleviated the impaired insulin resistance and ameliorated the responses of ITT and HOMA-IR in MS rats. Addition- ally, telmisartan significantly attenuated the increased adipose mass and reduced increased plasma TC and TG concentrations that may be due to modification of adipocyte 
A
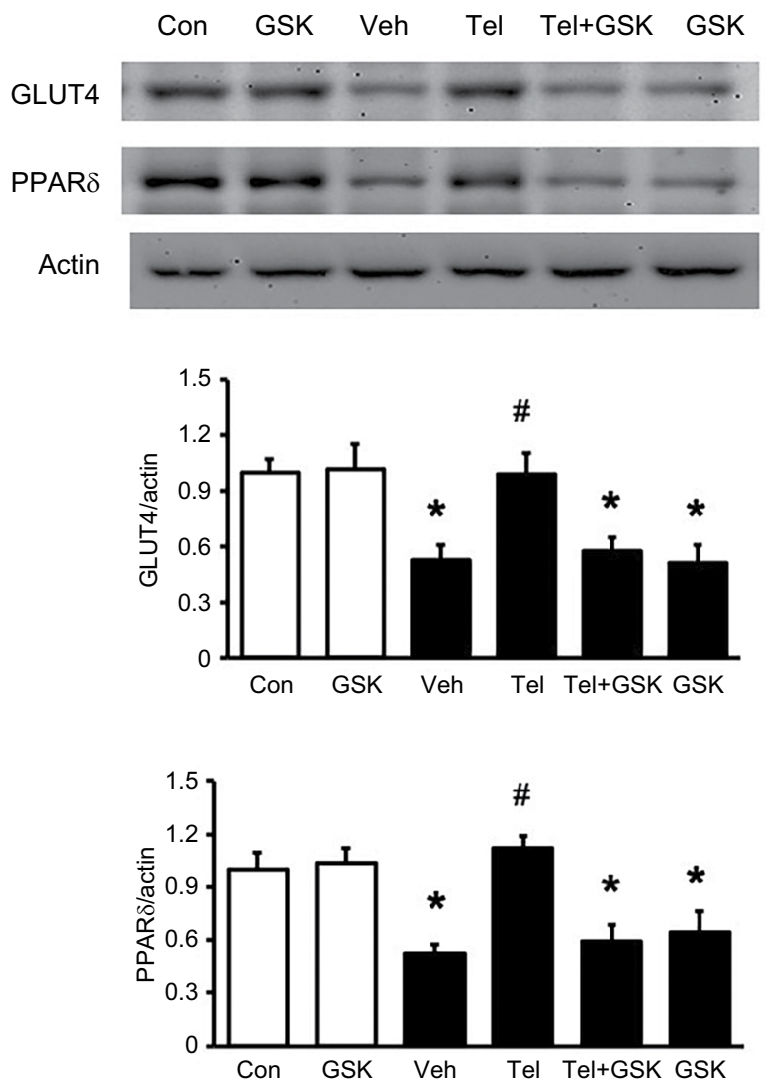

B
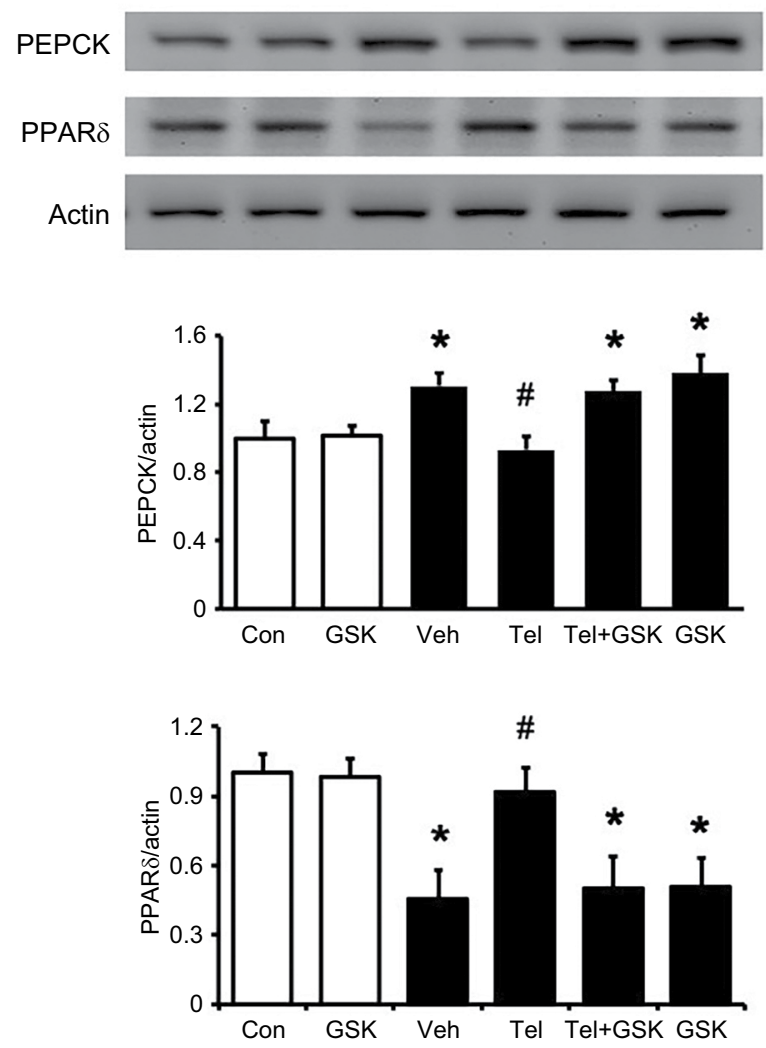

Figure 4 Effects of the PPAR $\delta$ antagonist GSK0660 on expressions of PPAR $\delta$ and related signals in skeletal muscle and liver isolated from the telmisartan-treated MS rats. Notes: (A) Representative immunoblots are shown in the upper part of the figure, and the relative expression levels of GLUT4 and PPAR $\delta$ in soleus muscle are indicated in the lower. (B) Representative immunoblots are shown in the upper part of the figure and the relative expression levels of PEPCK and PPAR $\delta$ in the liver are indicated in the lower. Con (white column), SD rats fed normal diet; Con+Tel (white column), SD rats fed normal diet and administered with telmisartan (8mg/kg); Veh (black column), MS rats received with vehicle; Veh+Tel (black column), MS rats received administered with telmisartan (8mg/kg) for 4 weeks; Veh+Tel+GSK (black column), MS rats received administered with telmisartan $(8 \mathrm{mg} / \mathrm{kg}$ ) for 4 weeks and GSK0660 were pretreated 30 minutes before telmisartan treatment; Veh+GSK (black column) MS rats received

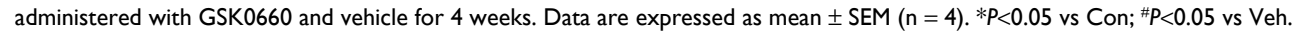

Abbreviations: Con, control; GSK, GSK0660; PEPCK, phosphoenolpyruvate carboxykinase; PPAR , peroxisome proliferator-activated receptor delta; MS, metabolic syndrome; SD, Sprague Dawley; Tel, telmisartan; Veh, vehicle.

biology and metabolism. ${ }^{36}$ This resulted in an increase of energy expenditure and decrease of dietary-induced obesity and/or accumulation of visceral fat. ${ }^{37,38}$ It is consistent with a previous report that telmisartan activates PPAR $\delta$ expression and reduces weight gain and HF-induced obesity. ${ }^{39}$ In this study, telmisartan produced a beneficial effect on hyperglycemia, insulin resistance, and lipid metabolism more effectively than losartan in the MS model, which is consistent with a previous report. ${ }^{40}$ In clinics, telmisartan showed effects on metabolic parameters to a greater degree than losartan in hypertensive patients with $\mathrm{MS} .{ }^{41}$ A largescale clinical study reported that hypertensive patients with type 2 diabetes had reduced plasma glucose and serum TG concentrations after 6 months' treatment with telmisartan compared with baseline values. ${ }^{42}$ Telmisartan may induce beneficial effects on MS by direct blockade of the AT1 receptor. Some studies have established that AT1 receptor stimulation by AT2 contributes to insulin resistance and its associated deleterious metabolic profile. ${ }^{43,44}$ Therefore, the AT1 receptor blockade ameliorates the disorders and partially explains the beneficial effects of telmisartan on insulin resistance. A recent study indicated that the prevention of weight gain by telmisartan is partly attributed to an Ang-(1-7)-dependent mechanism. ${ }^{45}$ However, it also indicated that lowering of BP in fructose-fed rats by the use of other antihypertensive drugs, such as calcium channel blockers, ${ }^{46,47}$ failed to show a metabolic impact, which suggests that telmisartan improved insulin resistance via BPindependent mechanisms. Moreover, our study showed that the beneficial effects of telmisartan on MS were markedly 
reversed by GSK0660 at the dose sufficient to block PPAR $\delta$. Therefore, telmisartan may activate PPAR $\delta$ to improve glucose and lipid metabolism and prevent the increase of insulin resistance induced by diet. ${ }^{48}$

Increase in insulin sensitivity is mainly induced by the enhancement of insulin signals. PPAR $\delta$ is the most abundant isoform among the three PPARs in skeletal muscle. ${ }^{49}$ Alternatively, Ang II (via AT receptor) is the predominant component of the RAS, which appears to be antagonistic to insulin action and contributes to insulin resistance..$^{50} \mathrm{Ang}$ II impairs the insulin-induced activation of IRS1 and Akt in addition to GLUT4 membrane translocation in skeletal muscle cells. ${ }^{51}$ Although it is an ARB, telmisartan could activate PPAR $\delta$ to increase the oxidative capacity and result in the usage of glucose or breakdown of fat. ${ }^{52}$ Insulin induces GLUT4 translocation to the cellular membrane to facilitate glucose uptake in skeletal muscle. Insulin resistance leads to defective PI3K/Akt signaling, reduced GLUT4 expression, and impaired insulin-stimulated glucose uptake. ${ }^{49}$ The present study demonstrated that telmisartan activates PPAR $\delta$ in the skeletal muscle of MS rats, which is consistent previous research. ${ }^{16}$ Moreover; telmisartan attenuated the increased expression of hepatic PEPCK in a dose-related manner. It has been documented that PPAR $\delta$ functions as a nuclear sensor of dietary fats, capable of modulating immune response through regulation of metabolic programs in the liver. ${ }^{53}$ Therefore, telmisartan could activate PPAR $\delta$ to alter peripheral insulin sensitivity and improve pancreatic $\beta$-cell function.

Elevated BP is associated with metabolic disorders. ${ }^{54} \mathrm{In}$ this study, HS intake was an important factor associated with the exacerbation of hypertension. ${ }^{55}$ Excessive salt intake may stimulate ROS production to increase the oxidative stress in various organs including muscle, liver and fat tissues in rats. ${ }^{56,57}$ HS diet also causes a decrease in the activity of circulating RAS to lower Ang II levels, which may induce the compensatory upregulation of AT receptors. ${ }^{58}$ Telmisartan, as a long-acting ARB, showed the antihypertensive effect more effectively than losartan, which is consistent with a previous report. ${ }^{59} \mathrm{~A} 3$-year study confirmed the advantage of telmisartan in controlling BP and reducing the risk of MS. ${ }^{60}$ Telmisartan may cause an AT receptor blockade to result in a fall of peripheral resistance ${ }^{59}$ or a PPAR-dependent increase in eNOS expression and activity. ${ }^{61}$ PPAR $\delta$ has been suggested as a potential therapeutic target in the treatment of hypertensive subjects with insulin resistance. We also confirmed that systemic blockade of PPAR $\delta$ seems to be associated with the elevation of BP in MS rats. Chronic PPAR $\delta$ agonist administration in the hypertensive rats induced a marked decrease in $\mathrm{BP} .{ }^{58}$ In addition, the PPAR $\delta$ agonist also induced the upregulation of hepatic lipid oxidation processes to suppress Ang II-induced dysfunctional adipogenesis and lipid accumulation. ${ }^{61}$

\section{Conclusion}

In summary, we have provided experimental evidence that telmisartan is effective in ameliorating hypertension, hyperinsulinemia, and hypertriglyceridemia through activation of PPAR $\delta$ in rats with MS. Therefore, the preclinical data support that treatment with telmisartan is suitable for managing patients with MS after clinical trials in the future.

\section{Author contributions}

All authors contributed to data analysis, drafting or revising the article, gave final approval of the version to be published, and agree to be accountable for all aspects of the work.

\section{Disclosure}

The authors report no conflict of interests in this work.

\section{References}

1. Srikanthan K, Feyh A, Visweshwar H, Shapiro JI, Sodhi K. Systematic review of metabolic syndrome biomarkers: a panel for early detection, management, and risk stratification in the West Virginian population. Int J Med Sci. 2016;13(1):25-38.

2. Misra A, Singhal N, Khurana L. Obesity, the metabolic syndrome, and type 2 diabetes in developing countries: role of dietary fats and oils. J Am Coll Nutr. 2010;29(3 Suppl):289S-301S.

3. Engin A. The definition and prevalence of obesity and metabolic syndrome. Adv Exp Med Biol. 2017;960:1-17.

4. Lakka HM, Laaksonen DE, Lakka TA, et al. The metabolic syndrome and total and cardiovascular disease mortality in middle-aged men. JAMA. 2002;288(21):2709-2716.

5. Hassannejad R, Kazemi I, Sadeghi M, et al. Longitudinal association of metabolic syndrome and dietary patterns: a 13-year prospective population-based cohort study. Nutr Metab Cardiovasc Dis. 2018;28(4):352-360.

6. Shankar E, Vykhovanets EV, Vykhovanets OV, et al. High-fat diet activates pro-inflammatory response in the prostate through association of Stat-3 and NF-kappaB. Prostate. 2012;72(3):233-243.

7. Ferrannini E, Natali A, Capaldo B, Lehtovirta M, Jacob S, Yki-Jarvinen H. Insulin resistance, hyperinsulinemia, and blood pressure: role of age and obesity. European Group for the Study of Insulin Resistance (EGIR). Hypertension. 1997;30(5):1144-1149.

8. Chen J, Gu D, Huang J, et al. Metabolic syndrome and salt sensitivity of blood pressure in non-diabetic people in China: a dietary intervention study. Lancet. 2009;373(9666):829-835.

9. Zhou MS, Schulman IH, Zeng Q. Link between the renin-angiotensin system and insulin resistance: implications for cardiovascular disease. Vasc Med. 2012;17(5):330-341.

10. Muller-Fielitz H, Hubel N, Mildner M, Vogt FM, Barkhausen J, Raasch W. Chronic blockade of angiotensin AT(1) receptors improves cardinal symptoms of metabolic syndrome in diet-induced obesity in rats. $\mathrm{Br} J$ Pharmacol. 2014;171(3):746-760. 
11. Tyagi S, Gupta P, Saini AS, Kaushal C, Sharma S. The peroxisome proliferator-activated receptor: a family of nuclear receptors role in various diseases. JAdv Pharm Technol Res. 2011;2(4):236-240.

12. Kramer DK, Al-Khalili L, Guigas B, Leng Y, Garcia-Roves PM, Krook A. Role of AMP kinase and PPARdelta in the regulation of lipid and glucose metabolism in human skeletal muscle. J Biol Chem. 2007;282(27):19313-19320.

13. Tanaka T, Yamamoto J, Iwasaki S, et al. Activation of peroxisome proliferator-activated receptor delta induces fatty acid beta-oxidation in skeletal muscle and attenuates metabolic syndrome. Proc Natl Acad Sci U S A. 2003;100(26):15924-15929.

14. Toral M, Gomez-Guzman M, Jimenez R, et al. Chronic peroxisome proliferator-activated receptorbeta/delta agonist GW0742 prevents hypertension, vascular inflammatory and oxidative status, and endothelial dysfunction in diet-induced obesity. J Hypertens. 2015;33(9):1831-1844.

15. Kakuta H, Sudoh K, Sasamata M, Yamagishi S. Telmisartan has the strongest binding affinity to angiotensin II type 1 receptor: comparison with other angiotensin II type 1 receptor blockers. Int J Clin Pharmacol Res. 2005;25(1):41-46.

16. Li L, Luo Z, Yu H, et al. Telmisartan improves insulin resistance of skeletal muscle through peroxisome proliferator-activated receptor-delta activation. Diabetes. 2013;62(3):762-774.

17. Shinohara T, Takahashi N, Abe I, et al. Telmisartan effectively improves insulin sensitivity in hypertensive patients with insulin resistance. Obes Res Clin Pract. 2011;5(4):e267-360.

18. Sanchez RA, Masnatta LD, Pesiney C, Fischer P, Ramirez AJ. Telmisartan improves insulin resistance in high renin nonmodulating saltsensitive hypertensives. J Hypertens. 2008;26(12):2393-2398.

19. Wong SK, Chin KY, Suhaimi FH, Fairus A, Ima-Nirwana S. Animal models of metabolic syndrome: a review. Nutr Metab (Lond). 2016;13:65.

20. Thirunavukkarasu V, Anitha Nandhini AT, Anuradha CV. Lipoic acid attenuates hypertension and improves insulin sensitivity, kallikrein activity and nitrite levels in high fructose-fed rats. J Comp Physiol B. 2004;174(8):587-592.

21. Ghibaudi L, Cook J, Farley C, van Heek M, Hwa JJ. Fat intake affects adiposity, comorbidity factors, and energy metabolism of spraguedawley rats. Obes Res. 2002;10(9):956-963.

22. Zhuhua Z, Zhiquan W, Zhen Y, et al. A novel mice model of metabolic syndrome: the high-fat-high-fructose diet-fed ICR mice. Exp Anim. 2015;64(4):435-442.

23. Panchal SK, Brown L. Rodent models for metabolic syndrome research. J Biomed Biotechnol. 2011;2011:351982.

24. Van den Bergh A, Vanderper A, Vangheluwe P, et al. Dyslipidaemia in type II diabetic mice does not aggravate contractile impairment but increases ventricular stiffness. Cardiovasc Res. 2008;77(2):371-379.

25. Mark AL, Shaffer RA, Correia ML, Morgan DA, Sigmund CD, Haynes WG. Contrasting blood pressure effects of obesity in leptin-deficient ob/ob mice and agouti yellow obese mice. J Hypertens. 1999;17(12 Pt 2):1949-1953.

26. Dong YF, Liu L, Kataoka K, et al. Aliskiren prevents cardiovascular complications and pancreatic injury in a mouse model of obesity and type 2 diabetes. Diabetologia. 2010;53(1):180-191.

27. Shin SJ, Lim JH, Chung S, et al. Peroxisome proliferator-activated receptor-alpha activator fenofibrate prevents high-fat diet-induced renal lipotoxicity in spontaneously hypertensive rats. Hypertens Res. 2009;32(10):835-845.

28. Geer EB, Islam J, Buettner C. Mechanisms of glucocorticoid-induced insulin resistance: focus on adipose tissue function and lipid metabolism. Endocrinol Metab Clin North Am. 2014;43(1):75-102.

29. Chen J, Huang XF, Shao R, Chen C, Deng C. Molecular mechanisms of antipsychotic drug-induced diabetes. Front Neurosci. 2017;11:643.

30. Dobrian AD, Schriver SD, Lynch T, Prewitt RL. Effect of salt on hypertension and oxidative stress in a rat model of diet-induced obesity. $\mathrm{Am}$ J Physiol Renal Physiol. 2003;285(4):F619-628.
31. Kaur G, Meena C. Amelioration of obesity, glucose intolerance, and oxidative stress in high-fat diet and low-dose streptozotocin-induced diabetic rats by combination consisting of "curcumin with piperine and quercetin”. ISRN Pharmacol. 2012;2012:957283.

32. Suman RK, Ray Mohanty I, Borde MK, Maheshwari U, Deshmukh YA. Development of an experimental model of diabetes co-existing with metabolic syndrome in rats. Adv Pharmacol Sci. 2016;2016:9463476.

33. LiYX, Cheng KC, Asakawa A, et al. Role of musclin in the pathogenesis of hypertension in rat. PLoS One. 2013;8(8):e72004.

34. Bowe JE, Franklin ZJ, Hauge-Evans AC, King AJ, Persaud SJ, Jones PM. Metabolic phenotyping guidelines: assessing glucose homeostasis in rodent models. J Endocrinol. 2014;222(3):G13-25.

35. Kuo SC, Chung HH, Huang CH, Cheng JT. Decrease of hyperglycemia by syringaldehyde in diabetic rats. Horm Metab Res. 2014;46(1):8-13.

36. Araki K, Masaki T, Katsuragi I, Tanaka K, Kakuma T, Yoshimatsu H. Telmisartan prevents obesity and increases the expression of uncoupling protein 1 in diet-induced obese mice. Hypertension. 2006;48(1):51-57.

37. Fujisaka S, Usui I, Kanatani Y, et al. Telmisartan improves insulin resistance and modulates adipose tissue macrophage polarization in high-fat-fed mice. Endocrinology. 2011;152(5):1789-1799.

38. Choi GJ, Kim HM, Kang H, Kim J. Effects of telmisartan on fat distribution: a meta-analysis of randomized controlled trials. Curr Med Res Opin. 2016;32(7):1303-1309.

39. He H, Yang D, Ma L, et al. Telmisartan prevents weight gain and obesity through activation of peroxisome proliferator-activated receptor-deltadependent pathways. Hypertension. 2010;55(4):869-879.

40. Khan AH, Imig JD. Telmisartan provides better renal protection than valsartan in a rat model of metabolic syndrome. Am J Hypertens. 2011;24(7):816-821.

41. Vitale C, Mercuro G, Castiglioni C, et al. Metabolic effect of telmisartan and losartan in hypertensive patients with metabolic syndrome. Cardiovasc Diabetol. 2005;4:6.

42. Michel MC, Bohner H, Koster J, Schafers R, Heemann U. Safety of telmisartan in patients with arterial hypertension: an open-label observational study. Drug Saf. 2004;27(5):335-344.

43. Cooper ME, Tikellis C, Thomas MC. Preventing diabetes in patients with hypertension: one more reason to block the renin-angiotensin system. J Hypertens Suppl. 2006;24(1):S57-63.

44. Jandeleit-Dahm KA, Tikellis C, Reid CM, Johnston CI, Cooper ME. Why blockade of the renin-angiotensin system reduces the incidence of new-onset diabetes. J Hypertens. 2005;23(3):463-473.

45. Schuchard J, Winkler M, Stolting I, et al. Lack of weight gain after angiotensin AT1 receptor blockade in diet-induced obesity is partly mediated by an angiotensin-(1-7)/Mas-dependent pathway. Br J Pharmacol. 2015;172(15):3764-3778.

46. Oron-Herman M, Sela BA, Rosenthal T. Risk reduction therapy for syndrome X: comparison of several treatments. Am J Hypertens. 2005;18(3):372-378.

47. Zorad S, Dou JT, Benicky J, et al. Long-term angiotensin II AT1 receptor inhibition produces adipose tissue hypotrophy accompanied by increased expression of adiponectin and PPARgamma. Eur J Pharmacol. 2006;552(1-3):112-122.

48. Chen ZC, Lee KS, Chen LJ, Wang LY, Niu HS, Cheng JT. Cardiac peroxisome proliferator-activated receptor delta (PPARdelta) as a new target for increased contractility without altering heart rate. PLoS One. 2013;8(5):e64229.

49. Angione AR, Jiang C, Pan D, Wang YX, Kuang S. PPARdelta regulates satellite cell proliferation and skeletal muscle regeneration. Skelet Muscle. 2011;1(1):33.

50. Perlstein TS, Henry RR, Mather KJ, et al. Effect of angiotensin receptor blockade on insulin sensitivity and endothelial function in abdominally obese hypertensive patients with impaired fasting glucose. Clin Sci (Lond). 2012;122(4):193-202.

51. Wei Y, Sowers JR, Nistala R, et al. Angiotensin II-induced NADPH oxidase activation impairs insulin signaling in skeletal muscle cells. $J$ Biol Chem. 2006;281(46):35137-35146. 
52. Wang YX, Zhang CL, Yu RT, et al. Regulation of muscle fiber type and running endurance by PPARdelta. PLoS Biol. 2004;2(10):e294.

53. Liu S, Hatano B, Zhao M, et al. Role of peroxisome proliferator-activated receptor $\{$ delta $\} /\{$ beta $\}$ in hepatic metabolic regulation. $J$ Biol Chem. 2011;286(2):1237-1247.

54. Hermida RC, Chayan L, Ayala DE, et al. Association of metabolic syndrome and blood pressure nondipping profile in untreated hypertension. Am J Hypertens. 2009;22(3):307-313.

55. Weinberger MH, Miller JZ, Luft FC, Grim CE, Fineberg NS. Definitions and characteristics of sodium sensitivity and blood pressure resistance. Hypertension. 1986;8(6 Pt 2):II127-134.

56. Uetake Y, Ikeda H, Irie R, et al. High-salt in addition to high-fat diet may enhance inflammation and fibrosis in liver steatosis induced by oxidative stress and dyslipidemia in mice. Lipids Health Dis. 2015;14:6.

57. Lenda DM, Boegehold MA. Effect of a high-salt diet on oxidant enzyme activity in skeletal muscle microcirculation. Am J Physiol Heart Circ Physiol. 2002;282(2):H395-402.
58. Nickenig G, Strehlow K, Roeling J, Zolk O, Knorr A, Bohm M. Salt induces vascular AT1 receptor overexpression in vitro and in vivo. Hypertension. 1998;31(6):1272-1277.

59. Sueta D, Koibuchi N, Hasegawa Y, et al. Telmisartan exerts sustained blood pressure control and reduces blood pressure variability in metabolic syndrome by inhibiting sympathetic activity. Am J Hypertens. 2014;27(12):1464-1471.

60. Peng J, Zhao Y, Zhang H, et al. Prevention of metabolic disorders with telmisartan and indapamide in a Chinese population with high-normal blood pressure. Hypertens Res. 2015;38(2):123-131.

61. Yuen CY, Wong WT, Tian XY, et al. Telmisartan inhibits vasoconstriction via PPARgamma-dependent expression and activation of endothelial nitric oxide synthase. Cardiovasc Res. 2011;90(1):122-129.
Diabetes, Metabolic Syndrome and Obesity: Targets and Therapy is an international, peer-reviewed open-access journal committed to the rapid publication of the latest laboratory and clinical findings in the fields of diabetes, metabolic syndrome and obesity research. Original research, review, case reports, hypothesis formation, expert opinion and commentaries are all considered for publication. The manuscript management system is completely online and includes a very quick and fair peer-review system, which is all easy to use. Visit http://www.dovepress.com/testimonials.php to read real quotes from published authors.

Submit your manuscript here: https://www.dovepress.com/diabetes-metabolic-syndrome-and-obesity-targets-and-therapy-journal 